\title{
Night-to-night variation of the oxygen desaturation index in sleep apnoea syndrome
}

\author{
I. Fietze*, K. Dingli ${ }^{\#}$, K. Diefenbach*, , N.J. Douglas, M. Glos*, M. Tallafuss*, W. Terhalle ${ }^{\uparrow,+}$, C. Witt*
}

Night-to-night variation of the oxygen desaturation index in sleep apnoea syndrome. I. Fietze, K. Dingli, K. Diefenbach, N.J. Douglas, M. Glos, M. Tallafuss, W. Terhalle, C. Witt. (C) ERS Journals Ltd 2004.

ABSTRACT: The current study investigated the night-to-night variability and diagnostic accuracy of the oxygen desaturation index (ODI), as measured by ambulatory monitoring, in the diagnosis of mild and moderate obstructive sleep apnoea-hypopnoea syndrome.

To assess the variability of the ODI, 35 patients were monitored at home during 7 consecutive nights by means of a portable recording device, the MESAM-IV $\AA$. The ODI variability factor and the influence of age, body mass index (BMI), alcohol, and body position were assessed. Furthermore, the diagnostic accuracy of the MESAM-IV was measured by comparison with polysomnographical outcomes in 18 patients.

During home recording, the median ODI was 10.9 (interquartile range: 5.8-16.1) across the patients. Although the reliability of the ODI was adequate, the probability of placing the patient in the wrong severity category (ODI $\leqslant 15$ or ODI $>15$ ) when only one single recording was taken is $14.4 \%$. ODI variability was not significantly influenced by age, BMI, time spent in a supine position, or mild dosages of alcohol. A good correlation was found between the apnoea-hypopnoea index and the ODI.

In conclusion, the findings suggest that the diagnostic accuracy of the MESAM-IV is strong, since the oxygen desaturation index is correlated with the apnoea-hypopnoea index. In most obstructive sleep apnoea-hypopnoea syndrome patients, oxygen desaturation index variability is rather small, and screening could be reliably based on single 1-night recordings.

Eur Respir J 2004; 24: 987-993.

\author{
*Sleep Laboratory, Respiratory Medicine, and \\ 'Institute of Clinical Pharmacology, Charité, \\ and ${ }^{+}$Clinical Pharmacogenomics, Berlin-Buch \\ GmbH, Berlin, Germany. "Respiratory Medi- \\ cine, University of Edinburgh, Edinburgh, UK. \\ Correspondence: I. Fietze \\ Centre of Sleep Medicine \\ Dept of Cardiology and Pulmonology \\ Charité - Universitätsmedizin Berlin \\ Campus Mitte \\ Humboldt University \\ Luisenstr. 13a \\ D-10117 Berlin \\ Germany \\ Fax: 4930450513906 \\ E-mail: ingo.fietze@charite.de
}

Keywords: Home monitoring oxygen desaturation index variability sleep apnoea diagnosis

Received: September 42003

Accepted after revision: August 122004
Diagnosis of the obstructive sleep apnoea-hypopnoea syndrome (OSAHS) is based on clinical symptoms [1] and on the demonstration of abnormal breathing during sleep, usually by in-hospital cardiorespiratory polysomnography (PSG) [2]. The limited number of sleep centres and beds, however, can result in long waiting lists for diagnosis. Homebased sleep studies have potential advantages in terms of decreased costs [3], convenience for the patients, and improved sleep quality. KINGSHOTT et al. [4] found better sleep efficiency, longer periods of rapid eye movement and slow-wave sleep, as well as significantly fewer arousals during home-based studies, when compared with in-laboratory studies. These findings suggest that home recordings are less likely to be influenced by first-night acclimatisation effects, and may more reliably reflect patients' actual sleep and breathing profiles.

The diagnosis of severe OSAHS is often based on symptoms and home-recording outcomes. The diagnosis of mild or moderate OSAHS may be a stepwise procedure, consisting of an initial home recording followed by in-hospital PSG [3]. There is controversy as to whether the use of homebased diagnosis affects continuous positive airway pressure (CPAP) compliance. WhitTLE et al. [3] used the portable EdenTrace system (EdenTec 3711; Nellcor, Eden Prairie, MN, USA) for home recording, and revealed no difference in nightly CPAP usage in comparison with PSG-diagnosed control patients over a period of 4-20 months. KRIEGER et al. [5] used the MESAM-IV device (MAP, Munich, Germany) and found significantly lower CPAP compliance during a 2-yr period in patients diagnosed at home, when compared with PSG-diagnosed control patients, although the same instructions and CPAP titration pathways were applied to all patients. Both studies covered symptomatic moderate and severe sleep apnoeics.

One possible interpretation of these findings is that MESAM-IV may overdiagnose sleep apnoeics who require CPAP treatment; an explanation supported by some studies [6]. This device is a validated [7], widely used, cost-effective, user-friendly portable system. Its main diagnostic criterion is the oxygen desaturation index (ODI), although the heartvariability index and the snoring index can also be assessed [6]. In a prospective study, RYAN et al. [8] assessed the diagnostic accuracy of the ODI criteria set by the British Thoracic Society (BTS), i.e. $\geqslant 15$ desaturations of $4 \%$ per hour in bed. An apnoea-hypopnoea index (AHI) $>15 \cdot \mathrm{h}^{-1}$, which was slept on PSG, was used as the objective measure of disease [9]. These authors found that the BTS criteria are highly specific when positive (100\% specificity), but may miss sleep apnoeics with no significant desaturations $(31 \%$ sensitivity). These findings suggest that an oximetry-based device is unlikely to overdiagnose patients, unless their ODIs vary significantly across nights. ODI variability (i.e. the variation of ODI throughout consecutive nights) is of clinical importance in mild-to-moderate sleep apnoeics, since it may affect the further management and treatment of these patients. 
The present study aims to examine night-to-night variability of ODI in symptomatic mild-to-moderate OSAHS patients. The study also tests the hypothesis that variability is influenced by age, body mass index (BMI), intake of small quantities of alcohol, and body position. The accuracy of the MESAM-IV device was tested by comparing its results with those of in-laboratory PSG.

\section{Methods}

\section{Study protocol}

The study had two parts. In part one (the variability study), seven sequential home recordings were taken from 35 patients to assess night-to-night variability of the parameters defined below. In part two (the comparison study), results from home-based recordings were compared with those of inlaboratory PSG in a subgroup of 18 patients. Within a period of 7 days, each patient was studied on the basis of the two methods (1 night each), in random order.

\section{Subjects}

A total of 35 patients (32 male, mean age $58 \pm 11$ yrs, mean BMI $26 \pm 3 \mathrm{~kg} \cdot \mathrm{m}^{-2}$ ) were recruited, with an ODI between 5 and $30 \cdot \mathrm{h}^{-1}$ in bed on the initial (baseline) home recording. All study patients had been referred to the sleep centre with possible OSAHS, and had experienced either self-reported daytime sleepiness (Epworth Sleepiness Scale $>10$ ) or two other major symptoms of OSAHS [1]. They provided written informed consent to participate in the study. The study had the approval of the local ethics committee.

In total, 18 out of the 35 patients (17 male, mean age $60 \pm 7 \mathrm{yrs}$, mean BMI $26 \pm 2 \mathrm{~kg} \cdot \mathrm{m}^{-2}$ ) underwent in-laboratory PSG.

Prior to each nocturnal recording, patients filled out a questionnaire on their daily alcohol consumption. According to their habits, patients were categorised into nonconsumers, occasional, and regular consumers of alcohol. Patients were excluded from the study if their daily alcohol consumption was $>0.5 \mathrm{~g}$ alcohol per $\mathrm{kg}$ body weight.

\section{Recordings}

The home recordings were performed with the portable MESAM-IV device (MAP) and consisted of: 1) finger pulse oximetry; 2) heart-rate detection, using a single-lead ECG; 3) an electric microphone; and 4) a body-position detector.

Since the MESAM-IV device does not record sleep, each patient received a sleep diary to record the light-out and lighton times, as well as awakenings and time spent awake. This information, along with the quality and regularity of the tracings, was used to mark the beginning and the end of each recording period and the intervals of awakenings.

PSG was performed with the computerised recording system SIDAS-GS (Singh Medical, Staefa, Switzerland) and consisted of: 1) four unipolar electroencephalography tracings, two central (C3A2, C4A1) and two occipital (O1A2, $\mathrm{O} 2 \mathrm{~A} 1$ ) electrodes, two outer canthi electrodes (LEOG, REOG), and a submental electromyography (EMG) electrode; 2) tibial EMG and a body-position detector; 3) threelead ECG; and 4) oronasal airflow detection by a thermistor sensor, and thoracoabdominal movement detection by two piezoelectric belts, a digital microphone, and a pulse oxymeter.
All diagnostic tools were employed by trained technicians. The portable device was placed into operation in the late afternoon, prior to each home monitoring. All recordings were carried out between 19:00 and 07:00 h, and their findings were assessed the following morning.

\section{Post-acquisition analysis}

All studies were scored manually in random order by the same observer who was blinded to subject name.

Home recordings. Respiratory events were scored when desaturations $\geqslant 3 \%$ occurred in the absence of moving artefacts and irrespective of co-existing changes in snoring or heart rate $[6,10]$. The number of scored desaturations divided by the estimated sleep duration (time in bed - waking times) results in the ODI. The ODI was the outcome measure of MESAM-IV recordings, which characterised disease severity. An ODI $\geqslant 5$ in the initial (baseline) home recording was the diagnostic threshold for inclusion of OSAHS patients [10, 11].

Disease severity was assessed after every nocturnal recording, which was based on two ODI categories: normal-to-mild $(\mathrm{ODI} \leqslant 15)$ and moderate-to-severe (ODI $>15)$. Patients were regarded as belonging to the category into which they had most often fallen during the 7-night study period. Subsequently, the probability of placing a patient into the wrong category was calculated if the classification was based on a single 1-night recording.

It was theoretically possible that a patient's most frequent ODI was $<5$ or $>30$; only upon occurrence during the initial (baseline) home recording did these ODI values lead to exclusion. The analysis was accordingly repeated with four ODI categories (normal: ODI $<5$; mild: $5 \leqslant$ ODI $\leqslant 15$; moderate: $15<\mathrm{ODI} \leqslant 30$; severe: ODI $>30$ ). The mean oxygen saturation and the mean lowest oxygen saturation were calculated across all patients and recordings.

Polysomnography. Sleep was scored according to RECHTSCHAFFEN and KALES criteria [12]. Apnoeas were defined as cessation of oronasal airflow lasting $\geqslant 10 \mathrm{~s}$. Hypopnoeas were defined as airflow reduction of $>50 \%$, compared with a 10 -s peak amplitude during the preceding $2 \mathrm{~min}$, lasting $\geqslant 10 \mathrm{~s}$ and associated with either oxygen desaturation of $\geqslant 3 \%$ or an arousal [2].

The extent of apnoea-hypopnoea was determined by two methods of calculation from each polysomnogram: first, by dividing the sum of the number of apnoeas (A) and hypopnoeas $(\mathrm{H})$ by the total sleep time (TST) $(\mathrm{AHI}=(\mathrm{A}+\mathrm{H}) /$ TST); and secondly, by dividing the total number of apnoeas and hypopnoeas by the hours in bed (TIB) $(\mathrm{A}+\mathrm{H} /$ $\mathrm{TIB}=(\mathrm{A}+\mathrm{H}) / \mathrm{TIB})$. According to international classifications, patients with $5 \leqslant \mathrm{AHI} \leqslant 15$ were classified as mild, and those with AHI $>15$ as moderate-to-severe sleep apnoeics [2].

\section{Statistical analysis}

Since the patient group was small and normal distribution could not be assumed, nonparametric tests were applied. A p-value $<0.05$ was accepted as statistically significant. Data were reported as mean $\pm \mathrm{SD}$, as well as median values and interquartile ranges (IQR).

The ODI variability factor of each patient was calculated as follows: individual ODI range divided by the median ODI of the 7 nights. Spearman's rank-order correlation analysis was performed to test reliability of ODI and oxygen saturation measurements. Additionally, the coefficient of reliability 
(ratio of mean variance of a single patient to global variance) was calculated.

Likewise, Spearman's rank-order correlation analysis was employed to assess the influence of age and BMI on the ODI variability factor. The influence of slight alcohol consumption on the ODI variability factor was determined using the Kruskal-Wallis nonparametric test.

The supine-position variability factor of each patient was calculated according to the ODI variability factor (range divided by the median). To investigate the influence of the time spent in a supine position on the ODI variability factor, the correlation between the ODI variability factor and the supine-position variability factor was analysed by applying Spearman's rank-order correlation. These analyses were performed with data from every individual patient and, separately, for the group of patients with less variability (ODI variability factor $<1$ ), and for the group of patients with higher variability (ODI variability factor $>1$ ).

The current authors assessed the diagnostic accuracy of MESAM-IV and the reproducibility of the patients' classifications into mild and moderate-to-severe OSAHS by applying kappa statistics, which evaluated the concordance between PSG and MESAM-IV recordings. Spearman's rank-order correlation analysis was employed to evaluate the correlation between the outcomes of the two methods.

\section{Results}

\section{Variability study}

All 35 patients completed the protocol. It was necessary to repeat two of the 245 home recordings due to failure of pulsoximetry (for a failure rate of $0.8 \%$ ). Two home recording nights were not included in the analysis (patient No. 16, nights 1 and 2) because the patient had a blocked nose and could not fall asleep.

Six patients $(17 \%)$ were $<50$ yrs old, $13(37 \%)$ were between 50 and $60 \mathrm{yrs}$ old, and $16(46 \%)$ were $>60 \mathrm{yrs}$ old. A total of $18 \%$ of the patients had a BMI $>30 \mathrm{~kg} \cdot \mathrm{m}^{-2}$. Alcohol consumption was evaluated in only 31 patients, since four did not entirely complete the questionnaires with respect to this. Three $(10 \%)$ drank alcohol regularly, $18(58 \%)$ were occasional consumers and 10 never drank.

Night-to-night variability. The median ODI for 7 nights was 10.9 (IQR: 5.8-16.1), with each patient contributing one data point to the median (fig. 1). The ODI variability factors of the 35 patients ranged $0.2-3.3$ (median: 1.0 , IQR: 0.8-1.4; table 1 ). A total of 18 patients $(51 \%)$ demonstrated an ODI variability factor $<1$, i.e. their ODI ranges did not exceed the respective median values over the 7 nights. In total, 17 patients $(49 \%)$ had an ODI variability factor $>1$.

Every nocturnal recording led to the patient's placement in one of two ODI-based categories (normal-to-mild, or moderate-to-severe). Patients were regarded as belonging to the category in which they had fallen most frequently during the 7-night study period. The probability of placing the patient in the wrong category was $14.4 \%$ if the decision was based on a single 1-night recording. In total, 18 patients $(51 \%)$ remained in the same category throughout, six patients changed once to another category, four patients changed category twice, and seven patients changed three times.

However, analysis revealed that the ODI values of eight of the patients were predominantly $<5$ and were, only occasionally, marginally elevated (ODI $\left.<10 \cdot \mathrm{h}^{-1}\right)$. No patient demonstrated predominantly severe OSAHS. When the analysis described previously was repeated with three categories of

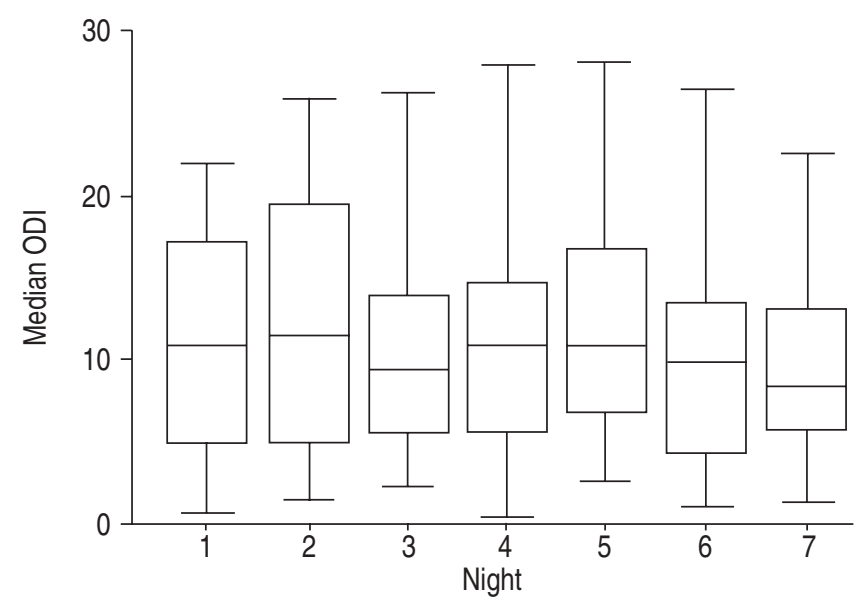

Fig. 1.- Median oxygen desaturation index (ODI) of 7 consecutive nights in 35 patients with mild-to-moderate obstructive sleep apnoeahypopnoea syndrome measured by ambulatory monitoring. Data are presented as a boxplot, where the black horizontal bar is the median, the box is the interquartile range (IQR) and the vertical lines extend to the smallest and largest observations within $1.5 \times \mathrm{IQR}$ of the quartiles.

severity (normal, mild, and moderate-to-severe), the probability of misclassifying the patient was $25.1 \%$ if the classification was based on a single 1-night recording. Nine patients $(26 \%)$ remained in the same category throughout all seven recordings. A total of 24 patients $(68 \%)$ changed between two categories, i.e. 10 patients $(28 \%)$ changed between normal and mild, and 14 patients $(40 \%)$ changed between mild and moderate-to-severe. Two patients $(6 \%)$ changed among all three categories.

The reliability coefficient of the ODI measurements over 7 consecutive nights was 0.72 , indicating that $72 \%$ of the variance is due to true variance of the ODI and that $28 \%$ of the variance depends on intra-individual variability. Spearman's rank-order correlation analysis disclosed that the ODI of all 7 nights correlated significantly $(p<0.01)$. The mean correlation coefficient was $0.73 \pm 0.05$, indicating a strong relationship.

Oxygen saturation. The median oxygen saturation over the 7 consecutive nights was $96.2 \%$ (IQR: 95.3-96.8\%), and the mean lowest saturation value was $90.8 \%$ (IQR: $89.1-92.2 \%$ ), with each patient contributing one data point to the median. The coefficient of reliability $(0.74)$ indicates that $74 \%$ of the variance is due to true variance of the median oxygen saturation, and that only $26 \%$ of the variance is due to intraindividual variability. Spearman's rank-order correlation analysis revealed that median oxygen saturation among all 7 nights correlated significantly $(\mathrm{p}<0.0001)$. The mean correlation coefficient was $0.70 \pm 0.06$, indicating a strong relationship.

Influence of age, body mass index and alcohol on the oxygen desaturation index variability factor. No correlation was found between the ODI variability factor and age $(\rho=-0.20$, $\mathrm{p}=0.28)$ or BMI $(\rho=-0.22, p=0.20)$ in the studied patients. The ODI variability factor was not influenced by the various levels of alcohol consumption in the 31 patients analysed $(p=0.54)$.

Body position and oxygen desaturation index variability factor. Six of the 35 patients were excluded from the analysis due to technical problems and failure in the bodyposition detector. The ODI variability factor did not correlate with the supine position variability factor $(\rho=-0.343, p=0.07)$. 
Table 1. - Oxygen desaturation indices (ODI) during 7 consecutive nights in 35 patients with mild and moderate obstructive sleep apnoea-hypopnoea syndrome

\begin{tabular}{|c|c|c|c|c|c|c|c|c|}
\hline Patient No. & ODI 1 & ODI 2 & ODI 3 & ODI 4 & ODI 5 & ODI 6 & ODI 7 & Variability \\
\hline 1 & 21.4 & 12.7 & 6.0 & 13.0 & 11.5 & 4.0 & 7.7 & 1.51 \\
\hline 2 & 15.8 & 9.3 & 13.7 & 10.8 & 12.1 & 26.5 & 20.2 & 1.26 \\
\hline 3 & 10.9 & 10.4 & 11.9 & 9.9 & 10.9 & 10.5 & 9.6 & 0.22 \\
\hline 4 & 14.0 & 8.0 & 9.3 & 5.6 & 18.2 & 9.7 & 8.2 & 1.36 \\
\hline 5 & 17.8 & 24.0 & 19.6 & 19.5 & 16.7 & 21.4 & 12.3 & 0.60 \\
\hline 6 & 17.1 & 25.4 & 26.1 & 5.4 & 9.9 & 7.0 & 12.3 & 1.69 \\
\hline 7 & 46.0 & 45.3 & 34.0 & 28.0 & 25.3 & 27.4 & 13.8 & 1.15 \\
\hline 8 & 10.6 & 13.7 & 14.0 & 10.9 & 15.0 & 11.0 & 10.9 & 0.40 \\
\hline 9 & 7.9 & 14.0 & 28.7 & 20.0 & 15.5 & 14.2 & 18.8 & 1.34 \\
\hline 10 & 14.3 & 18.6 & 10.5 & 11.5 & 18.2 & 10.8 & 10.7 & 0.70 \\
\hline 11 & 13.8 & 19.5 & 8.9 & 11.9 & 15.8 & 16.1 & 7.8 & 0.85 \\
\hline 12 & 13.3 & 6.5 & 5.8 & 13.1 & 11.4 & 8.7 & 13.2 & 0.66 \\
\hline 13 & 41.5 & 20.7 & 27.9 & 26.2 & 27.0 & 16.2 & 15.7 & 0.98 \\
\hline 14 & 21.6 & 22.7 & 29.8 & 31.9 & 28.2 & 12.9 & 14.3 & 0.83 \\
\hline 15 & 5.0 & 13.5 & 6.7 & 10.9 & 5.2 & 7.2 & 5.9 & 1.27 \\
\hline 16 & $21.0^{\#}$ & $42.2^{\#}$ & 7.4 & 2.1 & 3.2 & 4.3 & 8.4 & 1.48 \\
\hline 17 & 18.8 & 14.9 & 12.3 & 12.3 & 24.4 & 19.8 & 22.2 & 0.64 \\
\hline 18 & 1.8 & 2.5 & 4.0 & 0.3 & 3.1 & 2.1 & 5.3 & 1.94 \\
\hline 19 & 10.0 & 9.5 & 5.9 & 8.5 & 5.7 & 9.8 & 9.3 & 0.46 \\
\hline 20 & 9.5 & 16.9 & 22.3 & 15.7 & 9.3 & 4.6 & 4.5 & 1.88 \\
\hline 21 & 5.6 & 1.8 & 5.3 & 2.5 & 6.9 & 2.8 & 11.3 & 1.80 \\
\hline 22 & 12.6 & 24.0 & 11.0 & 18.3 & 18.8 & 25.4 & 22.6 & 0.77 \\
\hline 23 & 2.7 & 4.3 & 3.9 & 2.7 & 5.1 & 2.5 & 6.3 & 0.98 \\
\hline 24 & 1.1 & 1.5 & 2.2 & 1.9 & 2.8 & 1.0 & 3.3 & 1.19 \\
\hline 25 & 5.9 & 5.0 & 3.0 & 3.5 & 3.2 & 3.3 & 4.5 & 0.83 \\
\hline 26 & 3.0 & 5.5 & 4.7 & 5.1 & 6.9 & 7.0 & 3.0 & 0.78 \\
\hline 27 & 4.7 & 4.0 & 4.7 & 8.3 & 4.2 & 4.5 & 3.6 & 1.05 \\
\hline 28 & 15.0 & 24.0 & 12.9 & 24.3 & 16.8 & 9.9 & 14.3 & 0.96 \\
\hline 29 & 18.1 & 25.8 & 18.7 & 31.9 & 22.9 & 20.1 & 12.8 & 0.95 \\
\hline 30 & 4.9 & 4.2 & 4.4 & 5.7 & 6.8 & 2.9 & 7.1 & 0.85 \\
\hline 31 & 3.6 & 3.1 & 13.7 & 13.5 & 7.1 & 12.1 & 4.9 & 1.51 \\
\hline 32 & 0.6 & 1.8 & 6.5 & 4.1 & 10.6 & 3.0 & 1.3 & 3.33 \\
\hline 33 & 21.9 & 16.8 & 10.3 & 7.7 & 6.9 & 10.0 & 8.3 & 1.50 \\
\hline 34 & 5.8 & 9.3 & 7.1 & 6.0 & 11.2 & 12.9 & 5.6 & 1.02 \\
\hline 35 & 3.9 & 7.1 & 5.2 & 8.9 & 6.5 & 4.6 & 7.2 & 0.76 \\
\hline
\end{tabular}

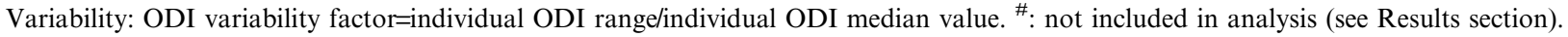

Furthermore, there was no correlation between the ODI and the time spent in supine position neither in the patient group as a whole $(\rho=0.008, p=0.91)$, nor in less variable patients (ODI variability factor $<1, \rho=0.068, p=0.50)$, nor in more variable patients (ODI variability factor $>1, \rho=0.14, \mathrm{p}=0.18$ ).

\section{Comparison study}

Sleep quality. The mean TST of the 18 patients was $6.4 \pm 0.8 \mathrm{~h}$, and mean sleep efficiency was $84.8 \pm 7.4 \%$.

Diagnostic accuracy. The mean AHI of the 18 patients was $17.3 \pm 11.8$, mean $\mathrm{A}+\mathrm{H} / \mathrm{TIB}$ was $14.7 \pm 10.2$, and mean ODI was 12.8 \pm 5.2 . The mean difference between the AHI and the ODI was $4.5 \pm 8.8$, and the mean difference between the $\mathrm{A}+\mathrm{H} / \mathrm{TIB}$ and the ODI was $1.8 \pm 7.6$. Spearman's rank-order correlation analysis revealed strong correlation between the ODI, and both the AHI $(\rho=0.78, p<0.0001)$ and the A+H/TIB $(\rho=0.77$, $\mathrm{p}<0.0001$; fig. 2). AHI and $\mathrm{A}+\mathrm{H} / \mathrm{TIB}$ did not lead to different classifications of patients, but correlated highly $(\rho=0.98$, $\mathrm{p}<0.0001)$. Thus, for further analysis, only the AHI was used.

A total of 13 of the 18 patients demonstrated homemeasured ODIs in the range of $5 \leqslant \mathrm{ODI} \leqslant 15$; nine of these 18 had an AHI in the same range (mean 9.7 \pm 2.7 ). One patient was borderline with an AHI of 4.7. In three patients, severity was underestimated with the MESAM-IV device; they demonstrated an AHI $>15$ on PSG (mean 29.2 \pm 12.2 ). Five of the patients had an ODI $>15$, of whom four had an AHI

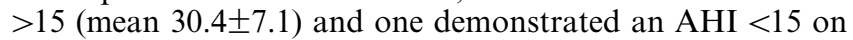
PSG.

Consequently, 13 of the 18 patients $(72.2 \%)$ were correctly classified by the portable device method (table 2). There was good agreement between MESAM-IV and PSG outcomes regarding patients' classifications into ODI-based and ADIbased severity groups, respectively $(\kappa$-coefficient $=0.51$, $\mathrm{p}=0.026$ ).

\section{Discussion}

The present study shows that the ODI is a sensitive indicator in the screening of mild and moderate sleep apnoeahypopnoea syndrome. In most patients, night-to-night variability of the ODI is not critical for correct screening of disease severity.

\section{Variability study}

The ODI did not change significantly during 1 week of repeated recordings in the majority of mild and moderate OSAHS patients, nor was it considerably influenced by age, BMI, nocturnal body position or alcohol habits. 

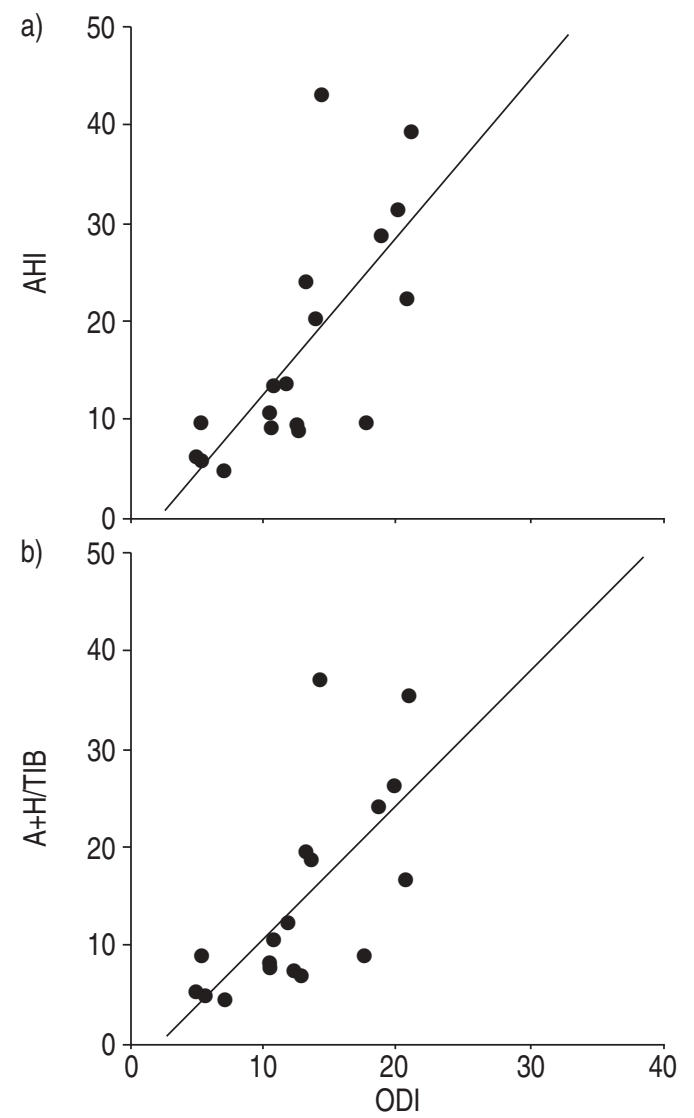

Fig. 2.-Correlation between the oxygen desaturation index (ODI), measured with an ambulatory monitoring system (MESAM-IV), and a) the apnoea-hypopnoea index (AHI) and b) the index of apnoeas and hypopnoeas per time in bed $(\mathrm{A}+\mathrm{H} / \mathrm{TIB})$, both measured by polysomnography in 18 patients with mild and moderate obstructive sleep apnoea-hypopnoea syndrome.

Table 2. - Outcomes of polysomnography and MESAM-IV in 18 patients with mild and moderate obstructive sleep apnoea-hypopnoea syndrome

\begin{tabular}{|c|c|c|c|c|c|c|}
\hline Patient No. & AHI & $\begin{array}{c}\text { AHI } \\
\text { severity }\end{array}$ & $\mathrm{A}+\mathrm{H} / \mathrm{TIB}$ & $\begin{array}{c}\mathrm{A}+\mathrm{H} / \mathrm{TIB} \\
\text { severity }\end{array}$ & ODI & $\begin{array}{c}\text { ODI } \\
\text { severity }\end{array}$ \\
\hline 1 & 31.4 & 2 & 26.4 & 2 & 20.0 & 2 \\
\hline 2 & 9.8 & 1 & 9.0 & 1 & 17.7 & 2 \\
\hline 3 & 9.0 & 1 & 7.3 & 1 & 12.4 & 1 \\
\hline 4 & 13.5 & 1 & 10.4 & 1 & 10.7 & 1 \\
\hline 5 & 24.1 & 2 & 19.7 & 2 & 13.2 & 1 \\
\hline 6 & 5.8 & 1 & 5.0 & 1 & 5.5 & 1 \\
\hline 7 & 20.4 & 2 & 18.7 & 2 & 13.8 & 1 \\
\hline 8 & 39.3 & 2 & 35.4 & 2 & 21.0 & 2 \\
\hline 9 & 13.8 & 1 & 12.4 & 1 & 11.8 & 1 \\
\hline 10 & 43.1 & 2 & 37.0 & 2 & 14.3 & 1 \\
\hline 11 & 28.8 & 2 & 24.0 & 2 & 18.7 & 2 \\
\hline 12 & 9.1 & 1 & 8.5 & 1 & 10.6 & 1 \\
\hline 13 & 22.2 & 2 & 16.7 & 2 & 20.7 & 2 \\
\hline 14 & 10.6 & 1 & 7.6 & 1 & 10.4 & 1 \\
\hline 15 & 4.7 & 0 & 4.5 & 0 & 7.0 & 1 \\
\hline 16 & 9.8 & 1 & 9.0 & 1 & 5.3 & 1 \\
\hline 17 & 9.6 & 1 & 6.8 & 1 & 12.6 & 1 \\
\hline 18 & 6.3 & 1 & 5.3 & 1 & 5.0 & 1 \\
\hline
\end{tabular}

AHI: apnoea-hypopnoea index per hour of sleep; A+H/TIB: apnoeas and hypopnoeas per hour in bed; ODI: oxygen desaturation index per hour in bed; severity groups: 0 ) $\mathrm{AHI}, \mathrm{A}+\mathrm{H} / \mathrm{TIB}$ or $\mathrm{ODI}<5,1) \mathrm{AHI} \geqslant 5$, $\mathrm{A}+\mathrm{H} / \mathrm{TIB}$ or ODI $\left.<15 \cdot \mathrm{h}^{-1}, 2\right) \mathrm{AHI}, \mathrm{A}+\mathrm{H} / \mathrm{TIB}$ or ODI $>15$.
It should be emphasised that the ODI threshold ( $>5$ ) was set a priori. It was based on previous study outcomes [10, 11], which indicated that the diagnostic threshold for pulse oximetry was correlated with sensitivity and inversely correlated with specificity. These findings were confirmed during the first part of the present study. With an ODI cut-off at $\geqslant 5$, none of the 35 patients would have been missed on the first of the 7 consecutive study nights. The ODI variability factor ranged $0.2-3.3$. In 17 of the 35 patients (48\%), the ODI variability factor was $>1$, i.e. the ODI range was greater than the median ODI in these patients. The probability of placing the patient in the wrong severity category when only one single recording was taken was $14.4 \%$ with two severity categories (normal-to-mild ODI $\leqslant 15$, and moderate-tosevere ODI $>15$ ) and $25.1 \%$ with three categories (normal ODI $<5$, mild $5 \leqslant$ ODI $\leqslant 15$, and moderate-to-severe ODI $>15$ ). A total of $94 \%$ of the patients either remained in the same diagnostic category throughout all 7 nights (nine patients), or changed between two diagnostic categories from mild-to-normal (10 patients) or from mild-to-moderate/ severe (14 patients).

The reliability of the ODI and of mean oxygen saturation measurements over the 7 nights was reasonably good, and the correlation among all 7 nights was strong. In general, the reliability coefficient or the correlation coefficient of a test should be $\geqslant 0.90$ if the test result is of consequence to a patient's treatment. However, ODI measurements with the MESAM-IV device are used only for screening; consequently, a coefficient of reliability of 0.72 and a mean coefficient of correlation of $0.73 \pm 0.05$ for the ODI are most probably adequate.

This finding concurs with earlier investigations reporting a highly consistent AHI in home studies [13]. These earlier findings disclosed that the ODI varied across all three diagnostic categories in only two patients $(6 \%)$ during the seven recordings. CHEDIAK et al. [14] observed that the AHI varied by $>10$ per hour of sleep in $32 \%$ of their patients during 2 PSG nights. This variation may be caused by alterations in the sleep pattern $[15,16]$, which are usually most pronounced in the first study night [17].

The present study aimed to identify the significance of night-to-night ODI variability, since such variability can affect the management and/or treatment compliance of mild and moderate symptomatic OSAHS patients. KRIEGER et al. [5] found decreased CPAP compliance in OSAHS patients whose diagnosis was based on clinical symptoms and on MESAM-IV recordings. The clinical symptoms and the analysis of the portable recordings were assessed qualitatively in a nonstandardised approach. Analysis of the recordings was based on recognition of a pattern that should dominate during $\geqslant 80 \%$ of the recording time to justify proceeding with treatment. No quantitative measure of symptoms or respiratory disturbance was established. Although these methodological issues render comparison to other study outcomes difficult, the present findings suggest that, in most patients, diagnostic accuracy of the ODI is not affected by its night-tonight variability.

\section{Age, body mass index, alcohol and body position}

Age, BMI and mild dosages of alcohol consumption of $\leqslant 0.5 \mathrm{~g}$ alcohol $\cdot \mathrm{kg}^{-1}$ did not affect the ODI variability factor. This concurs with findings of BERRY et al. [18], who reported that $0.5 \mathrm{~g}$ alcohol $\cdot \mathrm{kg}^{-1}$ did not notably change breathing patterns during sleep. In contrast, moderate doses of alcohol $\left(0.5-1 \mathrm{~g} \cdot \mathrm{kg}^{-1}\right)$ increased the AHI without influencing apnoea length or oxygen desaturation [19]. 
Time spent in a supine position did not significantly affect the ODI variability factor. It is well known that body position and the severity of an apnoeic event (duration and oxygen desaturation) are related in some patients [20]; however, the influence on ODI night-to-night variability was evidently insignificant in the current patient group. Nevertheless, when the MESAM-IV device is used, posture, snoring and heartrate variability remain important parameters for decisions on further diagnosis and for determination of appropriate therapy.

\section{Comparison study}

The good correlation between ODI and AHI suggests that both indices are accurate measures of the mild and moderate OSAHS. Since the correlation between the AHI per hour of sleep and $A+H / T I B$ was high, only the AHI was used for comparison with the ODI. Based on the selected cut-off levels (ODI $\geqslant 5$ on MESAM-IV, AHI $\geqslant 5$ on PSG), only one of the 18 home recordings $(6 \%)$ was false positive, with its borderline AHI of 4.5. None of the patients was classified as falsely negative (table 2). This indicates $100 \%$ sensitivity of an ODI $>5$ as diagnostic cut-off. A total of 13 of the 18 patients $(72 \%)$ were correctly classified by the portable device. It underestimated severity in three patients and overestimated in two. The three underestimated patients were classified as mild sleep apnoeics by the MESAM-IV device, and had an AHI $\geqslant 15$ per hour of sleep on PSG. Accordingly, outcomes of MESAM-IV measurements were in good concordance with those of PSG. The present findings suggest that only $28 \%$ of symptomatic mild and moderate-to-severe sleep apnoeics should undergo diagnostic PSG, following MESAM-IV recordings at home, for the purpose of evaluating disease severity. ESNAOLA et al. [7] compared PSG and simultaneously performed MESAM-IV recordings in 150 patients with suspected obstructive sleep apnoea. They found that the need for PSG was reduced by $75 \%$. The percentages determined by other groups are higher $[3,21]$. This is due to differences in the portable devices and the diagnostic thresholds used for PSG and for the portable studies [22].

With an ODI cut-off at $\geqslant 15$, three patients $(17 \%)$ would have been missed by the MESAM-IV device and one patient $(6 \%)$ would have been wrongly classified as being positive (sensitivity: $57 \%$, specificity: $91 \%$ ), which is in agreement with the findings of RYAN et al. [9]. A low ODI threshold augments sensitivity at the expense of specificity. However, therapy decisions should not be based on a single figure derived from an overnight home study, but, instead, should be interpreted in the context of the patient's clinical symptoms. The numerical diagnostic threshold is less important if the approach to diagnosis is more flexible.

\section{Study limitations}

Limitations of this study include the venue of the studies and the number of subjects studied. The purpose of the comparison study was to assess the accuracy of the ODI in mild-to-moderate sleep apnoeics. Had the study been limited to synchronous laboratory-based MESAM-IV versus PSG testing, the current authors would not have had a comparison with standard in-laboratory PSG, a particularly important aspect, since there are sleep-quality differences between recordings at home and in-laboratory [4]. The latter may have biased the comparison towards greater variance between ODI and AHI. It is believed that 18 patients are sufficient to demonstrate the broad agreement and the limitations of this approach, although acceptance of more subjects may have been desirable for this aim of the study. The main section of the study, however, with the larger number of patients, was intended to determine ODI variability in the patients' own environment. The current authors believe that the data obtained are adequate for firm conclusions relating to this objective.

\section{Conclusion}

To the current authors' knowledge, this is the first study to examine the diagnostic accuracy and night-to-night variation of the oxygen desaturation index in mild and moderate obstructive sleep apnoea-hypopnoea syndrome patients. The present findings suggest that the diagnostic accuracy of the MESAM-IV is good, as reflected by the strong correlation between the apnoea-hypopnoea index and the oxygen desaturation index. In most patients, oxygen desaturation index night-to-night variability was small, and screening could have been reliably based on a single 1-night measurement. Oxygen desaturation index night-to-night variability in patients with mild and moderate obstructive sleep apnoeahypopnoea syndrome was not significantly influenced by age, body mass index, time spent in a supine position, or small quantities of alcohol.

\section{References}

1. Whyte KF, Allen MB, Jeffrey A, Gould GA, Douglas NJ. Clinical features of the sleep apnoea/hypopnoea syndrome. Q J Med 1989; 72: 659-666.

2. American Academy of Sleep Medicine Task Force Report. Sleep-related breathing disorders in adults: recommendations for syndrome definition and measurement techniques in clinical research. Sleep 1999; 22: 667-689.

3. Whittle AT, Finch SP, Mortimore IL, MacKay TW, Douglas NJ. Use of home sleep studies for diagnosis of the sleep apnoea/hypopnoea syndrome. Thorax 1997; 52: 10681073.

4. Kingshott RN, Douglas NJ. The effect of in-laboratory polysomnography on sleep and objective daytime sleepiness. Sleep 2000; 23: 1109-1113.

5. Krieger J, Sforza E, Petiau C, Weiss T. Simplified diagnostic procedure for obstructive sleep apnoea syndrome: lower subsequent compliance with CPAP. Eur Respir J 1998; 12: 776-779.

6. Stoohs R, Guilleminault C. MESAM 4: an ambulatory device for detection of patients at risk for obstructive sleep apnoea syndrome (OSAS). Chest 1992; 101: 1221-1227.

7. Esnaola S, Duran J, Infante-Rivard C, Rubio R, Fernandez A. Diagnostic accuracy of a portable recording device (MESAM IV) in suspected obstructive sleep apnoea. Eur Respir J 1996; 9: 2597-2605.

8. British Thoracic Society. Facilities for the diagnosis and treatment of abnormal breathing during sleep including nocturnal ventilation. BTS News 1990; 5: 7-10.

9. Ryan PJ, Hilton MF, Boldy DAR, et al. Validation of British Thoracic Society guidelines for the diagnosis of the sleep apnoea/hypopnoea syndrome: can polysomnography be avoided? Thorax 1995; 50: 972-975.

10. Stoohs R, Bingham A, Itoi A, Guilleminault C, Dement W. Sleep and sleep-disordered breathing in commercial longhaul truck drivers. Chest 1995; 107: 1275-1282.

11. Douglas NJ, Thomas S, Jan MH. Clinical value of polysomnography. Lancet 1992; 339: 347-350.

12. Rechtschaffen A, Kales A. A manual of standardised terminology: techniques and scoring systems for sleep stages of human subjects. Washington DC, National Institutes of Health, 1968 (publication No. 204).

13. Wittig RM, Pomaker A, Zorick FJ, et al. Night-to-night 
consistency of apnoeas during sleep. Am Rev Respir Dis 1984; 129: 244-246.

14. Chediak AD, Acevedo-Crespo JC, Seiden DJ, et al. Nightly variability in the indices of sleep-disordered breathing in men being evaluated for impotence with consecutive night polysomnograms. Sleep 1996; 19: 589-592.

15. Dingli K, Fietze I, Asimakopoulos T, Quispe-Bravo S, Witt C, Douglas NJ. Arousability in sleep apnoea/hypopnoea syndrome patients. Eur Respir $J$ 2002; 20: 733-740.

16. Mosko SS, Dickel MJ, Ashurst J. Night-to-night variability in sleep apnoea and sleep-related periodic leg movements in the elderly. Sleep 1988; 11: 340-348.

17. Toussaint M, Luthringer R, Schaltenbrand N, et al. Firstnight effect in normal subjects and psychiatric inpatients. Sleep 1995; 18: 463-469.

18. Berry RB, Desa MM, Light RW. Effects of ethanol on efficacy of nCPAP as a treatment for OSA. Chest 1991; 99: 339-343.

19. Scanlan MF, Roebuck PJ, Little PJ, et al. Effect of moderate alcohol upon obstructive sleep apnoea. Eur Respir J 2000; 16 : 909-913.

20. Oksenberg A, Khamaysi I, Silverberg DS. Apnoea characteristics across the night in severe obstructive sleep apnoea: influence of body posture. Eur Respir J 2001; 18: 340-346.

21. Dingli K, Coleman EL, Vennelle M, et al. Evaluation of a portable device for diagnosing the sleep apnoea/hypopnoea syndrome. Eur Respir J 2003; 21: 253-259.

22. Redline S, Kapur VK, Sanders MH, et al. Effects of varying approaches for identifying respiratory disturbances on sleep apnoea assessment. Am J Respir Crit Care Med 2000; 161: 369-374. 